\title{
Manej o de Populações de Plantas Daninhas Resistentes aos Herbicidas InIBIDORES dA ACETOLACTATO SINTASE ${ }^{1}$
}

\author{
Management of Weed Populations Resistant to ALS-Inhibitor Herbicides
}

\author{
MONQUERO, P.A. ${ }^{2}$ e CHRISTOFFOLETI, P.J. ${ }^{3}$
}

\begin{abstract}
RESUMO - Nas áreas produtoras de soja da região central do Brasil é comum a infestação da planta daninha picão-preto (constituída de uma mistura das espécies Bidens pilosa e Bidens subalternans). Da mesma forma, o caruru (Amaranthus quitensis) é infestante da cultura da soja na Argentina. Estas plantas daninhas são controladas normalmente por diversos herbicidas; dentre os mais utilizados encontram-se os inibidores da acetolactato sintase (ALS). O uso intensivo e repetitivo destes herbicidas em áreas cultivadas com soja no município de São Gabriel do Oeste (MS-Brasil) e nas províncias de Córdoba e Tucumã (Argentina) selecionou populações resistentes destas plantas daninhas. Assim, com o objetivo de estudar alternativas de manejo dessas populações resistentes, foram desenvolvidos ensaios em condições de campo e casa de vegetação, por meio da aplicação de herbicidas inibidores da ALS (chlorimuron-ethyl e imazethapyr) e com mecanismos de ação alternativos; como inibidores da protoporfirinogênio oxidase (PROTOX), que participa da biossíntese de porfirina e tetrapirroles (lactofen e fomesafen) e inibidores do fotossistema II (bentazon). O ensaio de campo foi instalado no município de São Gabriel do Oeste-MS, Brasil, onde se suspeitava que a população de picãopreto da área fosse resistente, pois os herbicidas inibidores da ALS aplicados nos últimos anos na área não apresentavam eficiência esperada e a área tinha um histórico de pelo menos oito anos de aplicação sucessiva destes herbicidas. Concluiu-se, deste ensaio, que os herbicidas chlorimuron-ethyl e imazethapyr, nas doses recomendadas, foram ineficientes no controle da população resistente, porém os herbicidas alternativos lactofen, fomesafen e bentazon, aplicados isoladamente ou em mistura com os inibidores da ALS, foram eficazes. No ensaio conduzido em casa de vegetação, onde foram utilizados picão-preto e caruru, provenientes das áreas com suspeita de resistência, bem como populações de áreas que não tinham histórico da aplicação de herbicidas inibidores da ALS, foram obtidos resultados que confirmaram a resistência destas populações e a eficácia dos herbicidas alternativos obtidos em condições de campo. Como conclusão geral da pesquisa, ressalta-se que as populações resistentes de picão-preto e caruru estudadas possuem resistência cruzada às sulfoniluréias e imidazolinonas, mas não possuem resistência múltipla aos herbicidas inibidores do fotossistema II e aos inibidores da PROTOX, sugerindo que estes herbicidas alternativos podem ser utilizados para prevenção e manejo da resistência aos herbicidas inibidores da ALS.
\end{abstract}

Palavras-chave: resistência, Bidens pilosa, Bidens subalternans, Amaranthus quitensis.

ABSTRACT - Soybean production areas of the central region of Brazil are commonly infested by the weed beggarticks (a mixture of the species Bidens pilosa and Bidens subalternans), similarly to soybean in Argentina, commonly infested by the redroot pigweed (Amaranthus quitensis). These weeds are usually controlled by several herbicides among which the most used are the ALS inhibitors. The intensive and repetitive use of these herbicides in São Gabriel do Oeste, MS - Brazil and in the provinces of Córdoba and Tucumã (Argentina) has selected resistant populations of these weeds. Therefore, the objective of this research was carry out

Recebido para publicação em 17/12/1999 e na forma revisada em 24/3/2000.

2 Eng.-Agrônoma. Mestranda em Fitotecnia pela ESALQ/USP, Bolsista FAPESP; ${ }^{3}$ Prof. Associado, Departamento de Produção Vegetal - ESALQ/USP, Caixa Postal 09, 13418-900 Piracicaba-SP, <pjchrist@ carpa.ciagri.usp.br>.

Planta Daninha, Viçosa-MG, v.19, n.1, p.67-74, 2001 
field and greenhouse experiments to study the management of these resistant populations, spraying as treatments the ALS-inhibitor herbicides chlorimuron-ethyl and imazethapyr and herbicides with alternative mechanism of action, protoporphyrinogen oxidase - PROTOX inhibitors (lactofen and fomesafen) and photosystem II inhibitor (bentazon). The field experiment was conducted at São Gabriel do Oeste, MS - Brazil, where the suspected resistant population of beggarticks has not been controlled by ALS-inhibitor herbicides, in the last years, which had been sprayed annually for, at least, eight years. It was concluded that the herbicides chlorimuronethyl and imazethapyr were not efficient in controlling the weed population at recommended rates. However, the herbicides with alternative mechanisms of action lactofen, fomesafen and bentazon, sprayed alone or in mixture with the ALS-inhibitor herbicides gave a good control of the weed. In the greenhouse experiment conducted with beggartick and redroot pigweed, whose seeds were collected from the suspected resistant sites, and seeds from a population that had never been sprayed with ALS-inhibitor herbicides, results similar to those obtained under field conditions were confirmed. Overall, it can be emphasized that the studied resistant populations of beggarticks and redroot pigweed are cross resistant to sufonylureas and imidazolinones, lacking, however, multiple resistance mechanisms to photosystem II inhibitors and PROTOX inhibitors, suggesting that these herbicides are good alternative for management of the resistant populations.

Key words: $\quad$ weed resistance, management, Bidens pilosa, Bidens subalternans, Amaranthus quitensis.

\section{INTRODUÇÃO}

A resistência de plantas daninhas aos herbicidas é uma resposta evolutiva destas espécies de plantas às práticas agrícolas. Após a descoberta da ação herbicídica do 2,4-D no controle de plantas daninhas, na década de 40, as indústrias químicas têm desenvolvido novas moléculas, as quais têm sido adotadas largamente na agricultura. Por causa do uso intensivo destes produtos, em muitas regiões, como único método de controle de plantas daninhas, ocorreu uma pressão de seleção sobre as populações de plantas daninhas, ocasionando o aparecimento de populações resistentes aos herbicidas. Recentemente, áreas agrícolas com ocorrência de populações resistentes têm aumentado grandemente, devido, principalmente, à introdução de novos grupos químicos de herbicidas, dentre eles sulfoniluréias, imidazolinonas, triazolopirimidinas e pirimidil tiobenzoatos. Esses produtos inibem a acetolactato sintase (ALS), também chamada de acetohydroxyacido synthase (AHAS), que catalisa a primeira reação na produção dos aminoácidos de cadeia ramificada valina, leucina e isoleucina.

O desenvolvimento da resistência das plantas daninhas aos herbicidas é influenciado por vários fatores, dentre os quais aqueles ligados ao produto químico, como os herbicidas altamente eficientes, que apresentam um único local de ação, com residual prolongado, e a utilização intensiva do mesmo herbicida ou de herbicidas com o mesmo mecanismo de ação, que aumentam a pressão de seleção do biótipo resistente. A biologia da planta daninha também pode influenciar a taxa na qual a resistência se desenvolve; por exemplo, as plantas daninhas de ciclo anual podem desenvolver resistência mais rapidamente do que as espécies bianuais ou perenes, pois maior número de gerações serão submetidas ao agente selecionador. A diversidade genética é de fundamental importância no desenvolvimento da resistência; algumas espécies possuem alta taxa natural de mutações gênicas conferindo resistência a uma classe de herbicidas antes mesmo que estes sejam aplicados no campo. Muitas vezes, a característica de resistência pode também ser disseminada através do pólen e das sementes, aumentando assim o fluxo gênico que confere resistência para áreas adjacentes.

Os herbicidas inibidores ALS são largamente utilizados em áreas agrícolas, devido à alta eficácia no controle de várias espécies de plantas daninhas, às baixas doses recomendadas, à baixa toxicidade aos mamíferos e à seletividade a várias culturas. Os herbicidas inibidores da ALS foram comercializados a partir de 1982 para o controle de plantas daninhas do tipo folha larga. Em 1987, foi registrado o primeiro caso de resistência com 
populações de Lactuca serriola, resistentes ao chlorsulfuron nos Estados Unidos (MallorySmith et al., 1990). Atualmente, há cerca de 53 espécies que apresentam biótipos resistentes aos grupos químicos inibidores da ALS (Heap, 1999).

É necessária a modificação de algumas práticas agrícolas de modo a prevenir ou retardar o estabelecimento da resistência em alguns biótipos de plantas daninhas (Gressel \& Segel, 1989). A rotação de culturas reduz o efeito das plantas daninhas fisiologicamente próximas da cultura e permite o uso de herbicidas alternativos, com diferentes mecanismos de ação, o que pode representar uma estratégia eficaz no manejo da resistência (Christoffoleti et al., 1994). Técnicas que reduzem o banco de sementes de plantas daninhas podem ser incorporadas na prática de rotação de culturas, como pastagem ou produção forrageira, períodos de pousio utilizando herbicidas não-seletivos ou cultivadores, utilização de adubos verdes e queima de resíduos de plantas daninhas após a colheita (Powles \& Holtum, 1994).

O manejo racional de herbicidas é uma prática bastante importante, e o uso de herbicidas sem nenhuma, ou com pouca, atividade residual no solo e a otimização de doses e número de aplicações reduzem a pressão de seleção, diminuindo os riscos de seleção de resistência de plantas aos herbicidas. Da mesma forma, a rotação de herbicidas com diferentes mecanismos de ação diminui consideravelmente a pressão de seleção para biótipos resistentes (Christoffoleti et al., 1994).

O uso de misturas de herbicidas para manejo e prevenção da resistência está baseado no fato de que os ingredientes ativos controlam eficientemente os dois biótipos da mesma espécie, ou seja, o biótipo resistente a um dos herbicidas é controlado pelo outro ingrediente ativo da mistura (Powles \& Holtum, 1994). É importante ressaltar que a mistura de herbicidas de diferentes mecanismos de ação como forma de manejo e prevenção de resistência é mais eficiente quando o sistema de reprodução da planta daninha é a autogamia, uma vez que a recombinação gênica de diferentes alelos que conferem resistência tem menor probabilidade de ocorrer em relação a plantas alógamas (Gould, 1995).
Outras alternativas para prevenção e manejo da resistência podem ser: cultivo de culturas mais competitivas, espaçamento mais adensado, controle biológico e uso de cobertura morta. É interessante manter um histórico de cada área da propriedade para se identificar a evolução da população de determinadas espécies, pois, normalmente, a ocorrência dos biótipos resistentes não pode ser detectada durante os primeiros anos de aplicação do agente selecionador, isto é, quando é percebida a falta de controle de uma espécie que tradicionalmente era controlada por certo herbicida, já decorreram diversos anos do início da seleção do biótipo resistente. Assim, as principais formas de manejo e prevenção da resistência estão todas relacionadas ao manejo integrado de plantas daninhas.

Esta pesquisa teve como objetivo a avaliação da sensibilidade de populações de uma mistura das espécies de plantas daninhas Bidens pilosa e Bidens subalternans aos herbicidas inibidores da ALS e a herbicidas com outros mecanismos de ação (herbicidas alternativos) em condições de campo. Também foram testados nesta pesquisa diferentes herbicidas para manejo de populações de Bidens pilosa/ Bidens subalternans (picão-preto) e de Amaranthus quitensis (caruru) com herbicidas inibidores da ALS em condições de casa de vegetação.

\section{MATERIAL E MÉTODOS}

\section{Experimento de campo}

O experimento foi instalado na Fazenda Ponte Vermelha, município de São Gabriel do Oeste, MS, no dia 22 de dezembro de 1997, em área cultivada com a cultura de soja, onde o manejo de plantas daninhas do tipo folha larga vinha sendo feito há pelo menos oito anos, por meio de herbicidas pertencentes aos grupos químicos das imidazolinonas (imazaquin e imazethapyr) e sulfoniluréias (chlorimuronethyl). Entre as plantas daninhas infestantes da área, o picão-preto (mistura de Bidens pilosa e Bidens subalternans) era a mais abundante, no entanto, nos últimos anos, os índices de controle obtidos tinham sido abaixo do normalmente esperado. Suspeitou-se, portanto, que na área onde foi instalado o experimento havia

Planta Daninha, Viçosa-MG, v.19, n.1, p.67-74, 2001 
uma população de picão-preto resistente aos herbicidas inibidores da ALS.

O delineamento experimental adotado foi de blocos ao acaso, com dez tratamentos e quatro repetições. As parcelas possuíam área útil de $24 \mathrm{~m}^{2}$, ou seja, oito linhas de soja espaçadas de $0,5 \mathrm{~m}$, por $6,0 \mathrm{~m}$ de comprimento, onde foram feitas as aplicações dos herbicidas testados.

Os tratamentos utilizados, as doses de ingrediente ativo e produto comercial por hectare estão descritos na Tabela 1. A aplicação foi realizada no dia 22 de dezembro de 1997, com pulverizador costal pressurizado com $\mathrm{CO}_{2}$, munido de oito bicos, com ponta XR 110 02, aplicando um volume de calda correspondente a $200 \mathrm{~L} \mathrm{ha}^{-1}$, com pressão de trabalho de 2,10 bar. As condições ambientais foram: temperatura de $29^{\circ} \mathrm{C}$, umidade relativa do ar no início da aplicação (8 h 15 min) de 56\% e no final da aplicação (10 horas) de 70\%. A população predominante no momento da aplicação era de picão-preto, constituído de uma mistura de Bidens pilosa e Bidens subalternans, com cerca de $85 \%$ de infestação no estádio de 4-6 folhas definitivas.

Tabela 1 - Descrição dos tratamentos utilizados no experimento instalado no município de São Gabriel do Oeste-MS

\begin{tabular}{|l|c|c|}
\hline \multicolumn{1}{|c|}{ Tratamento } & g i.a. ha ${ }^{-1}$ & g ou L p.c. ha $^{-1}$ \\
\hline 1. chlorimuron-ethyl & 20,0 & 80 \\
2. chlorimuron-ethyl + lactofen & $12,5+120$ & $50+0,5$ \\
3. chlorimuron-ethyl + fomesafen & $12,5+150$ & $50+0,6$ \\
4. chlorimuron ethyl + bentazon & $12,5+384$ & $50+0,8$ \\
5. chlorimuron ethyl + imazethapyr & $12,5+50$ & $50+0,5$ \\
6. lactofen & 192 & 0,8 \\
7. fomesafen & 225 & 0,9 \\
8. bentazon & 480 & 1,0 \\
9. imazethapyr & 100 & 1,0 \\
10. testemunha & - & - \\
\hline
\end{tabular}

i.a. $=$ ingrediente ativo; p.c. $=$ produto comercial.

- Adjuvante Agral foi adicionado nos tratamentos 1, 3, 4, 7 e 8, na dose de $0,2 \% \mathrm{v} / \mathrm{v}$.

Foi avaliado o controle das plantas aos 14 e 21 dias após a aplicação dos herbicidas (DAA). Para quantificar o controle do picão-preto por meio dos herbicidas, utilizou-se escala visual, atribuindo a nota 0 para nenhum controle e
100 para controle total, comparados à testemunha.

Os dados foram submetidos à análise de variância para obtenção do valor de $\mathrm{F}$, sendo este significativo; as médias foram comparadas através do teste de Tukey. Para análise estatística, os resultados das quatro repetições foram transformados para $\operatorname{arcsen} \sqrt{\% / 100}$.

\section{Casa de vegetação}

O experimento foi conduzido em casa de vegetação do Departamento de Produção Vegetal da Escola Superior de Agricultura "Luiz de Queiroz”, Universidade de São Paulo, Piracicaba, São Paulo. As sementes de picão-preto resistentes (R) aos herbicidas inibidores da ALS foram coletadas a partir de plantas que sobreviveram aos tratamentos com herbicidas deste mecanismo de ação (chlorimuron-ethyl e imazethapyr) do experimento de campo conduzido em São Gabriel do Oeste-MS, Brasil. As sementes de caruru foram coletadas na Argentina, a partir de plantas remanescentes da aplicação de herbicidas inibidores da ALS em experimento conduzido pelo pesquisador Hugo Bergener, do INTA. Segundo informações deste pesquisador, nessa área o manejo de plantas daninhas do tipo folha larga tinha sido feito por diversos anos consecutivos (pelo menos oito anos) com herbicidas inibidores da ALS, e o controle da planta daninha caruru nos últimos anos tem sido falho, embora em populações normais estes herbicidas tenham apresentado controle satisfatório. Outras amostras de sementes consideradas suscetiveis (S) de picão-preto e caruru foram coletadas de áreas que nunca receberam pulverizações com os herbicidas inibidores da ALS, em São Gabriel do Oeste (Brasil) e Argentina, respectivamente.

As sementes $\mathrm{R}$ e $\mathrm{S}$ de ambas as espécies foram semeadas separadamente, em copos plásticos de $200 \mathrm{ml}$, contendo substrato à base de solo e material orgânico, na proporção 3:1. Os vasos foram irrigados de acordo com a necessidade, para manter umidade satisfatória, por meio de um sistema automático de irrigação por aspersão instalado na casa de vegetação, fazendo-se duas irrigações diárias de $10 \mathrm{~mm}$. Vasos com plantas uniformes e vigorosas com quatro folhas definitivas foram selecionados para os tratamentos com herbicidas. 
Os tratamentos herbicidas utilizados estão descritos na Tabela 2, e a aplicação dos herbicidas foi feita através de pulverizador constituído de um bico com ponta do tipo leque $80.03 \mathrm{E}$, pressurizado com ar comprimido a 2,10 bar. Foi avaliado o controle das plantas aos 14 dias após a aplicação dos herbicidas. Utilizou-se uma escala visual que variava de 0 para nenhum controle e $100 \%$ para controle total. Aos 16 dias foi determinada, após secagem em estufa, a biomassa seca.

Tabela 2 - Tratamentos utilizados no experimento de casa de vegetação, em picão-preto e caruru R e S

\begin{tabular}{|c|c|c|}
\hline Tratamento & i.a. g ha $^{-1}$ & p.c. g ou L ha ${ }^{-1}$ \\
\hline 1. Chlorimuron-ethyl & 20 & 80 \\
\hline 2. Chlorimuron-ethyl + fomesafen & $20+250$ & $80+1,0$ \\
\hline 3. Chlorimuron-ethyl + lactofen & $20+192$ & $50+0,8$ \\
\hline 4. Chlorimuron-ethyl + imazethapyr & $20+100$ & $50+1,0$ \\
\hline 5. Chlorimuron-ethyl + bentazon & $20+480$ & $50+1,5$ \\
\hline 6. Lactofen & 192 & 0,8 \\
\hline 7. Fomesafen & 250 & 1,0 \\
\hline 8. Bentazon & 480 & 1,5 \\
\hline 9. Testemunha & - & - \\
\hline
\end{tabular}

Os resultados foram analisados através da análise de variância, para obtenção do valor de F, sendo este significativo; as médias foram comparadas por meio do teste de Tukey. Para análise estatística, os resultados foram transformados para arcsen $\sqrt{\% / 100}$.

\section{RESULTADOS E DISCUSSÃO}

\section{Campo}

Os tratamentos com os herbicidas inibidores da ALS isolados (chlorimuron-ethyl ou imazethapyr) ou em mistura entre si não controlaram picão-preto satisfatoriamente; o tratamento com o herbicida imazethapyr apresentou resultado estatisticamente semelhante ao da testemunha (Tabela 3).

Os resultados obtidos comprovam que, nesta área cultivada com soja, houve a seleção de um biótipo de picão-preto resistente aos herbicidas inibidores da ALS. A aplicação destes herbicidas no controle das plantas daninhas do tipo folha larga durante oito anos aumentou a freqüência de alelos resistentes a estes herbicidas, ultrapassando a freqüência crítica, ou seja, os índices de controle são considerados insatisfatórios.

Tabela 3 - Controle da planta daninha picão-preto, em experimento de campo aos 14 e 21 dias após a aplicação dos herbicidas (DAA). São Gabriel do Oeste, MS, 1998

\begin{tabular}{|c|c|c|}
\hline \multirow{2}{*}{ Tratamento } & \multicolumn{2}{|c|}{ DAA } \\
\hline & 14 & 21 \\
\hline 1 - Chlorimuron ethyl & $42,5 \mathrm{c}$ & $21,6 \mathrm{c}$ \\
\hline 2 - Chlorimuron ethyl + lactofen & $53,8 \mathrm{bc}$ & $63,8 \mathrm{~b}$ \\
\hline 3 - Chlorimuron ethyl + fomesafen & $72,5 \mathrm{a}$ & $72,5 \mathrm{ab}$ \\
\hline 4 - Chlorimuron ethyl + bentazon & $47,5 \mathrm{c}$ & $67,8 b$ \\
\hline 5 - Chlorimuron ethyl + imazethapyr & $37,5 \mathrm{c}$ & $17,2 \mathrm{c}$ \\
\hline 6 - Lactofen & $80,0 \mathrm{a}$ & $80,5 \mathrm{ab}$ \\
\hline 7 - Fomesafen & $82,6 \mathrm{a}$ & $85,3 \mathrm{a}$ \\
\hline 8 - Bentazon & $66,7 a b$ & $72,6 a b$ \\
\hline 9 - Imazethapyr & $0,3 \mathrm{~d}$ & $0,0 \mathrm{~d}$ \\
\hline 10 - Testemunha & $0,0 \mathrm{~d}$ & $0,0 \mathrm{~d}$ \\
\hline $\mathrm{F}$ & $107,16^{* *}$ & $127,33 * *$ \\
\hline CV $(\%)$ & 10,71 & 11,18 \\
\hline
\end{tabular}

$* *=$ teste $\mathrm{F}$ significativo a $1 \%$ de probabilidade.

Obs.: Médias acompanhadas de letras iguais na mesma coluna não diferem estatisticamente entre si pelo teste de Tukey a $5 \%$ de probabilidade.

Os herbicidas inibidores da ALS, quando em mistura com herbicidas alternativos fomesafen, lactofen (inibidores da PROTOX) e bentazon (inibidores do fotossistema II) -, apresentaram maior eficiência no controle de picão-preto, porém esses resultados foram menores que o esperado, pois a dose utilizada no experimento foi menor do que a recomendada, de acordo com Rodrigues \& Almeida (1995).

Deve ser utilizado sempre, nas misturas, herbicidas com diferentes mecanismos de ação, pois, se um biótipo for resistente a um dos produtos da mistura, poderá ser eliminado pelo outro ingrediente ativo da mistura (Powles \& Holtum, 1994). Essa estratégia pode retardar o aparecimento de resistência, uma vez que, se a freqüência inicial de alelos para a resistência é de $10^{-6}$ para cada um dos herbicidas 
envolvidos na mistura, a chance de que uma única planta tenha alelos múltiplos de resistência para ambos os herbicidas da mistura será de aproximadamente $10^{-12}$.

É importante ressaltar que a mistura de herbicidas de diferentes mecanismos de ação é útil para plantas autógamas, pois, no caso de polinização cruzada, plantas que apresentam alelos individuais conferindo resistência poderão se cruzar e, assim, aumentar a probabilidade de progênie resistente a ambos os herbicidas (Gould, 1995).

Os herbicidas alternativos, utilizados isoladamente, controlaram a população resistente de Bidens pilosa/Bidens subalternans, evidenciando, assim, a importância de se evitar o uso contínuo, na mesma área, dos mesmos herbicidas ou de herbicidas com o mesmo mecanismo de ação, por meio da rotação de herbicidas e da rotação de culturas. Christoffoleti et al. (1994) afirmam que a rotação de culturas permite, também, a utilização de diferentes herbicidas, colaborando, dessa forma, na redução da pressão de seleção sobre o agroecossistema.

O Comitê Brasileiro de Resistência de Plantas aos Herbicidas (CBRPH) recomenda, para prevenção e manejo da resistência, o manejo integrado de plantas daninhas, mantendose um ambiente desfavorável para estas plantas, mediante o emprego isolado ou combinado dos métodos biológicos, culturais, mecânicos, químicos e físicos de controle. O objetivo básico é a redução da infestação de plantas daninhas para niveis que não interfiram na produtividade econômica das culturas.

\section{Casa de vegetação}

Os herbicidas inibidores da ALS, imazethapyr e chlorimuron-ethyl, quando aplicados sozinhos ou em mistura entre si, não foram eficientes, como esperado, no controle das populações resistentes das duas espécies, porém controlaram satisfatoriamente as populações suscetíveis (Tabelas 4 e 5). De acordo com Lorenzi (1991), os herbicidas chlorimuronethyl e imazethapyr são recomendados para o controle destas plantas daninhas em pósemergência precoce na cultura da soja, sendo largamente utilizados e apresentando controle adequado em populações não submetidas à pressão de seleção.

Tabela 4 - Controle aos 14 dias após a aplicação dos herbicidas (DAA) e produção de biomassa seca dos biótipos resistentes e suscetíveis de Bidens pilosa/Bidens subalternans. Piracicaba, 1999

\begin{tabular}{|c|c|c|c|c|}
\hline \multirow{3}{*}{ Tratamento } & \multicolumn{2}{|c|}{ Biótipo Resistente } & \multicolumn{2}{|c|}{ Biótipo Suscetível } \\
\hline & $14 \mathrm{DAA}$ & Biomassa seca & 14 DAA & Biomassa seca \\
\hline & $\%$ controle & $\mathrm{g} /$ planta & $\%$ controle & $\mathrm{g} /$ planta \\
\hline 1 - Chlorimuron-ethyl & $39,10 \mathrm{~b}$ & $0,135 \mathrm{ab}$ & $75,05 \mathrm{a}$ & $0,032 \mathrm{~b}$ \\
\hline 2 - Chlorimuron-ethyl + fomesafen & $78,60 \mathrm{a}$ & $0,017 \mathrm{~d}$ & $83,53 \mathrm{a}$ & $0,012 \mathrm{~b}$ \\
\hline 3 - Chlorimuron-ethyl + lactofen & $86,76 \mathrm{a}$ & $0,010 \mathrm{~d}$ & $90,00 \mathrm{a}$ & $0,010 \mathrm{~b}$ \\
\hline 4 - Chlorimuron-ethyl + imazethapyr & $31,39 \mathrm{~b}$ & $0,09 \mathrm{bc}$ & 76,17 a & $0,015 \mathrm{~b}$ \\
\hline 5 - Chlorimuron-ethyl + bentazon & $84,30 \mathrm{a}$ & $0,030 \mathrm{~cd}$ & $84,30 \mathrm{a}$ & $0,015 \mathrm{~b}$ \\
\hline 6 - Lactofen & 90,00 a & $0,012 \mathrm{~d}$ & 86,76 a & $0,010 \mathrm{~b}$ \\
\hline 7 - Fomesafen & 82,16 a & $0,0125 \mathrm{~d}$ & 78,93 a & $0,020 \mathrm{~b}$ \\
\hline 8 - Bentazon & 78,75 a & $0,020 \mathrm{~d}$ & $78,75 \mathrm{a}$ & $0,015 \mathrm{~b}$ \\
\hline 9 - Testemunha & $0,00 \mathrm{~b}$ & $0,175 \mathrm{a}$ & $0,00 \mathrm{~b}$ & $0,152 \mathrm{a}$ \\
\hline $\mathrm{F}$ & $35,89 *$ & $32,05 *$ & $42,05^{*}$ & $23,49 *$ \\
\hline $\mathrm{CV}(\%)$ & 20,37 & 36,04 & 17,22 & 41,70 \\
\hline
\end{tabular}

Obs.: Médias acompanhadas de letras iguais na mesma coluna não diferem entre si pelo teste de Tukey a 5\% de probabilidade.

$*$ = teste $\mathrm{F}$ significativo a $1 \%$ de probabilidade. 
Tabela 5 - Controle aos 14 dias após a aplicação dos herbicidas (DAA) e produção de biomassa seca dos biótipos resistentes e suscetíveis de Amaranthus quitensis. Piracicaba, 1999

\begin{tabular}{|c|c|c|c|c|}
\hline \multirow{3}{*}{ Tratamento } & \multicolumn{2}{|c|}{ Biótipo Resistente } & \multicolumn{2}{|c|}{ Biótipo Suscetível } \\
\hline & $14 \mathrm{DAA}$ & Biomassa seca & $14 \mathrm{DAA}$ & Biomassa seca \\
\hline & $\%$ controle & $\mathrm{g} /$ planta & $\%$ controle & g/planta \\
\hline 1 - Chlorimuron-ethyl & $11,09 \mathrm{~b}$ & $0,165 \mathrm{~b}$ & $78,93 \mathrm{a}$ & $0,015 \mathrm{~b}$ \\
\hline 2 - Chlorimuron-ethyl + fomesafen & $90,00 \mathrm{a}$ & $0,020 \mathrm{~d}$ & $74,14 \mathrm{a}$ & $0,017 \mathrm{~b}$ \\
\hline 3 - Chlorimuron-ethyl + lactofen & $83,53 \mathrm{a}$ & $0,012 \mathrm{~d}$ & $90,00 \mathrm{a}$ & $0,015 \mathrm{~b}$ \\
\hline 4 - Chlorimuron-ethyl + imazethapyr & $18,14 b$ & $0,102 \mathrm{bc}$ & $68,59 \mathrm{a}$ & $0,017 \mathrm{~b}$ \\
\hline 5 - Chlorimuron-ethyl + bentazon & $63,80 \mathrm{a}$ & $0,027 \mathrm{~cd}$ & 65,46 a & $0,037 \mathrm{~b}$ \\
\hline 6 - Lactofen & $85,54 \mathrm{a}$ & $0,010 \mathrm{~d}$ & 82,16 a & $0,012 \mathrm{~b}$ \\
\hline 7 - Fomesafen & $78,75 \mathrm{a}$ & $0,015 \mathrm{~d}$ & $79,69 \mathrm{a}$ & $0,017 \mathrm{~b}$ \\
\hline 8 - Bentazon & $58,04 \mathrm{ab}$ & $0,032 \mathrm{~cd}$ & 58,45 a & $0,050 \mathrm{~b}$ \\
\hline 9 - Testemunha & $0,00 \mathrm{~b}$ & $0,290 \mathrm{a}$ & $0,00 \mathrm{~b}$ & $0,297 \mathrm{a}$ \\
\hline $\mathrm{F}$ & $20,15^{*}$ & $41,87 *$ & $24,12 *$ & $53,45^{*}$ \\
\hline $\mathrm{CV}(\%)$ & 18,49 & 25,79 & 26,70 & 44,18 \\
\hline
\end{tabular}

Obs.: Médias acompanhadas de letras iguais na mesma coluna não diferem entre si pelo teste de Tukey a 5\% de probabilidade.

$*=$ teste $\mathrm{F}$ significativo a $1 \%$ de probabilidade.

Quando se aplicou a mistura de herbicidas inibidores da ALS com os herbicidas alternativos, como lactofen, fomesafen e bentazon, o controle se mostrou eficiente tanto nas populações suscetíveis como nas resistentes. Do mesmo modo, os herbicidas alternativos utilizados sozinhos foram satisfatórios no controle e reduziram substancialmente a produção de biomassa em ambos os biótipos das duas espécies estudadas. Embora não diferindo estatisticamente, os tratamentos com lactofen, chlorimuron-ethyl + lactofen e chlorimuronethyl + bentazon foram eficientes no controle de picão-preto e caruru (Tabelas 4 e 5).

De acordo com Powles \& Holtum (1994), quando os biótipos apresentam resistência a apenas uma classe de herbicida, o sucesso no manejo da resistência pode ser alcançado através de herbicidas alternativos. Contudo, esta é uma estratégia de sucesso a curto prazo, uma vez que, se forem utilizados intensivamente, há o risco de se desenvolver resistência também para estes herbicidas alternativos.

A resistência é um fato crescente em todas as áreas agrícolas e pode ocorrer com qualquer classe de herbicidas, desde que incorretamente manejado; na maioria dos casos, o aparecimento dos biótipos resistentes está associado ao uso repetido do mesmo herbicida ou de herbicidas com o mesmo mecanismo de ação, sempre associado à monocultura. É necessário, portanto, o treinamento e a divulgação de estratégias que previnam e retardem o aparecimento de resistência, como o uso de herbicidas alternativos, a mistura ou seqüência de herbicidas com diferentes mecanismos de ação e o manejo de herbicidas.

\section{LITERATURA CITADA}

CHRISTOFFOLETI, P.J.; VICTORIA FILHO, R., SILVA, C.B. Resistência de plantas daninhas aos herbicidas. Planta Daninha, v.12, n.1, p.13-20, 1994.

GOULD, F. Comparisons between resistance management strategies for insects and weeds. Weed Technol., v.9, n.4, p.830-839, 1995.

GRESSEL, J.; SEGEL, L.A. Modeling the effectiveness of herbicide rotation and mixtures strategies to delay or preclude resistance. Weed Technol., v.4, p.186-198. 1989.

HEAP, I. International survey of herbicide resistant weeds. On line. (Internet.www.weedscience.com, $6^{\text {th }}$ October. 1999)

LORENZI, H.J. Plantas daninhas do Brasil. 2.ed. Nova Odessa: Plantarum, 1991. 440p. 1991.

Planta Daninha, Viçosa-MG, v.19, n.1, p.67-74, 2001 
MALLORY-SMITH, C.A.; THILL, D.C., DIAL, M.J. Identification of sulfonylurea herbicide resistant prickly lettuce (Lactuca serriola). Weed Technol., v.4, p.787-790, 1990.

POWLES, S.B.; HOWAT, P.D. Herbicide resistance weeds in Australia. Weed Technol., v.4, p.178185, 1990.
POWLES, S.; HOLTUM, J. Herbicide resistance in plants: biology and biochemistry. New York: CRC Press, 1994. 353p

RODRIGUES, B.N.; ALMEIDA, F.S. Guia de herbicidas, 3.ed. Londrina: Edição dos Autores, 1995. 675p. 